\title{
Gradient Index Devices for Terahertz Spoof Surface Plasmon Polaritons
}

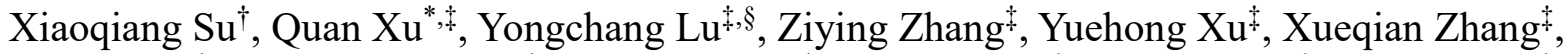 \\ Yanfeng $\mathrm{Li}^{\ddagger}$, Chunmei Ouyang ${ }^{\ddagger}$, Fusheng Deng ${ }^{\dagger}$, Yanhong Liu ${ }^{\dagger}$, Lijuan Dong ${ }^{\dagger}$, Yunlong Shi ${ }^{\dagger}$, \\ Xixiang Zhang ${ }^{\S}$, Jiaguang Han ${ }^{*}$, , and Weili Zhang ${ }^{*}, *$ \\ $\dagger$ Institute of Solid State Physics and College of Physics and Electronic Science, Shanxi Province \\ Key Laboratory of Microstructure Electromagnetic Functional Materials, Shanxi Datong \\ University, Datong 037009, China \\ Center for Terahertz Waves and College of Precision Instrument and Optoelectronics \\ Engineering, Key Laboratory of Optoelectronic Information Technology (Ministry of Education \\ of China), Tianjin University, Tianjin 300072, China \\ §Physical Science and Engineering Division King Abdullah University of Science and \\ Technology Thuwal 23955-6900, Saudi Arabia \\ * School of Electrical and Computer Engineering Oklahoma State University Stillwater, \\ Oklahoma 74078, USA
}

\begin{abstract}
Terahertz electromagnetic fields can be well confined and propagate at structured metal surfaces, known as spoof surface plasmon polaritons (SSPPs). Recent demonstrations of terahertz SSPP waveguides have attracted much attention for their potential in developing ultra-compact plasmonic circuits. However, further progress has been hampered by the lack of complex and highperformance devices which can flexibly manipulate the propagation of terahertz SSPPs. Here, we demonstrate several terahertz gradient index SSPP devices using metallic pillar structures. The devices are realized by engineering the geometric parameters of each metallic pillar to achieve the desired local mode index for the terahertz SSPPs. The performance of these SSPP devices is experimentally characterized using scanning near-field terahertz microscopy. The versatility of the presented design scheme would provide a platform for the design and fabrication of high-
\end{abstract}


performance plasmonic devices, especially cascadable ones in practical applications.

KEY WORDS: spoof surface plasmon polaritons, terahertz devices, plasmonics, gradient-index optics, surface waves

\section{TOC GRAPHIC}

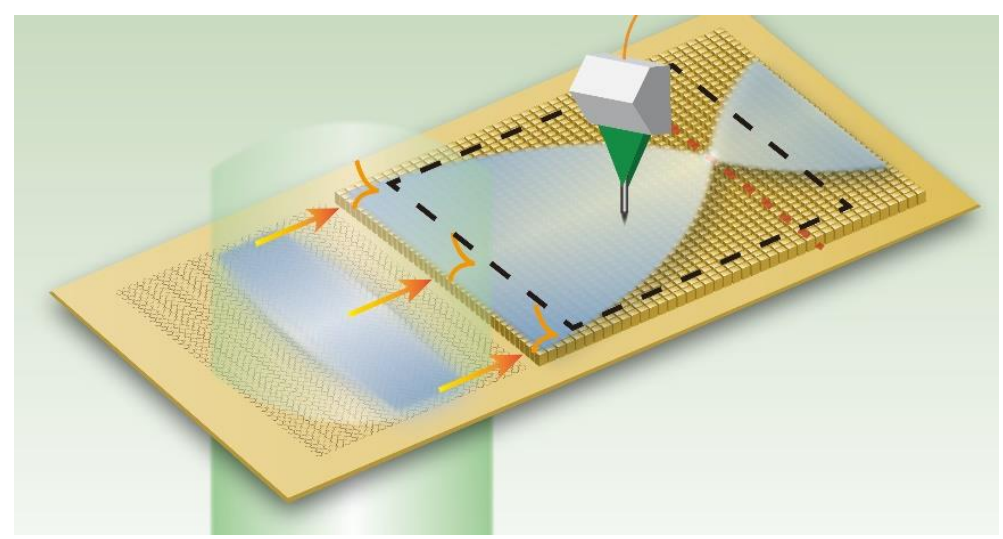




\section{Introduction}

In order to support the ever-increasing demand in both research and applications of terahertz $(\mathrm{THz})$ radiation and to bring it to the next level, miniaturized and multifunctional on-chip $\mathrm{THz}$ systems are one of the frontier research areas and being actively explored. ${ }^{1-4}$ On the technological level, this requires novel devices to manipulate $\mathrm{THz}$ electromagnetic fields at the deep subwavelength scale. Surface plasmon polaritons (SPPs) arise from the collective electron density oscillation coupled to external electromagnetic waves. ${ }^{5}$ Due to strong field confinement and large field enhancement of the SPPs, compact plasmonic devices such as lenses, ${ }^{6-8}$ logic gates, ${ }^{9,10}$ and switchable launchers ${ }^{11-14}$ have been demonstrated at the visible and near-infrared frequencies, emerging as ideal candidates for integrated on-chip plasmonic circuits. ${ }^{15-17}$ In contrast, high confinement of the SPPs at flat metal surfaces can hardly be achieved in the THz regime due to the fact that metals behave close to perfect electric conductors (PECs). ${ }^{5}$ To solve this problem, Pendry et al. proposed that surface waves confined on a structured PEC surface mimic the familiar SPPs at optical frequencies and are known as spoof SPPs (SSPPs). ${ }^{18}$ In the last decade, SSPPs have been developed from the metamaterial concept to practical applications, ${ }^{19-21}$ including waveguiding, ${ }^{22-24}$ lasing, ${ }^{25}$ sensing, ${ }^{26,27}$ filtering, ${ }^{28,29}$ etc. However, most of the related works were carried out at microwave frequencies, while the experimental studies at $\mathrm{THz}$ frequencies were mainly focused on simple waveguides, ${ }^{30-34}$ and more complex plasmonic devices still remain a challenge.

Inspired by the gradient index (GRIN) optics and transformation optics in free-space, great efforts have been made in realizing high performance plasmonic devices by variable refractive index structures. ${ }^{35-39}$ Different from critical transformation optics that requires a spatial variation of anisotropic materials and extreme values for both the permittivity and permeability, ${ }^{40}$ the 
structures used in these works were isotropic and non-magnetic by applying a prudent transformation scheme, i.e., solely modulating the mode index distributions could sufficiently manipulate the propagation of SPPs in a prescribed manner. ${ }^{41,42}$ Following this approach, complex devices for the manipulation of THz SSPPs can also be achieved once the effective mode index can be freely controlled by proper structures. In this letter, we demonstrate that subwavelength metallic pillars offer a powerful solution capable of controlling the local effective index of the $\mathrm{THz}$ SSPPs, paving the way for practical plasmonic device design. By using semiconductor processing technologies and scanning near-field terahertz microscopy (SNTM), proof-of-concept GRIN lens along with three integrated devices for THz SSPPs are fabricated and characterized. The proposed methodology illustrates an implementation combining strong field confinement with flexible propagation tailoring in the same platform.

\section{Results}

The basic unit cell for index manipulation is schematically shown in Figure 1a, which is a subwavelength metallic pillar on a metallic slab. Such a unit cell can support the propagation of SSPPs and the corresponding dispersion could be adjusted by changing the height $h$, width $a$, and period $p$ of the pillar. Since metals fundamentally show the characteristics of a PEC in THz regime, the skin depth is generally less than $100 \mathrm{~nm}$. In a realistic design, instead of directly constructing pure metallic pillars, we choose to etch silicon pillars using deep reactive ion etching and then coat them with 200-nm-thick Au films (see Section 1 of the Supporting Information for the fabrication details). Such Au-coated silicon pillars could be equivalently treated as pure metallic pillars since the THz fields can hardly interact with the silicon materials. The height and period of all the pillars were fixed as $h=70 \mu \mathrm{m}$ and $p=80 \mu \mathrm{m}$, respectively, to ensure easy fabrication. Figure $1 \mathrm{~b}$ 
illustrates the dispersion relations for the fundamental mode in the first Brillouin zone for pillars with different lateral widths, which were obtained by using the eigen-mode solver of the commercial software CST Microwave Studio. In the eigen-mode simulation, the entire unit cell was set as PECs, which is approximately valid for metals in the THz regimes (see Section 2 of the Supporting Information for more details about simulation). The large wavevectors around 0.73 THz showing slow light features, means that the SSPPs can be well confined on metallic pillars. Due to the well-known trade-off between the field confinement and loss, the propagation loss of such THz SSPPs on metallic pillars increases significantly compared with that on metal/air interface. ${ }^{4,22}$ For instance, the attenuation length of a metallic-pillar-based THz spoof waveguide has been experimentally measured as $9.2 \mathrm{~mm}$, which is about 3 orders of magnitude smaller than that of a metal/air interface. ${ }^{33}$ From the dispersion relations, the effective mode index of the unit cells can be calculated as: ${ }^{43}$

$$
n_{e f f}(f)=k(f) / k_{0}(f),
$$

where $f$ is the frequency and $k_{0}$ is the free-space wave number. According to Equation (1), the effective mode index at $0.73 \mathrm{THz}$ for varying lateral widths $a$ from $34 \mu \mathrm{m}$ to $76 \mu \mathrm{m}$ with a step of $2 \mu \mathrm{m}$ is retrieved, as shown in Figure 1c. Clearly the index can be flexibly modified in the range about 1 - 1.5. Once the relationship between the THz SSPP effective mode index and the pillar width is known, the width profiles for different GRIN plasmonic devices or transformation plasmonic devices can be readily interpolated according to the desired index profiles. 
(a)

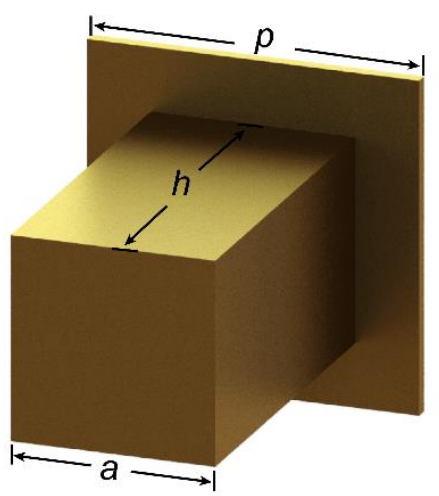

(c)

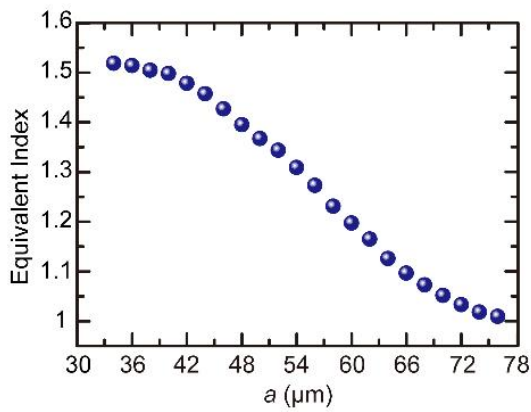

(b)

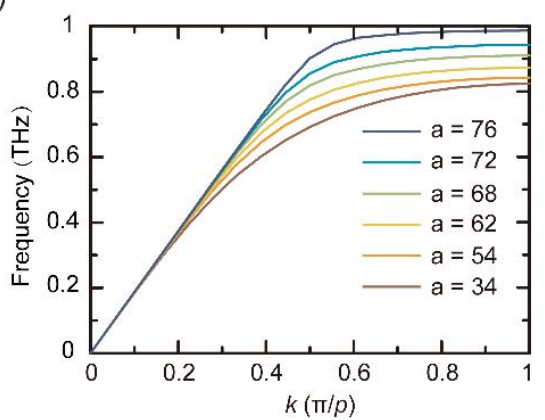

(d)

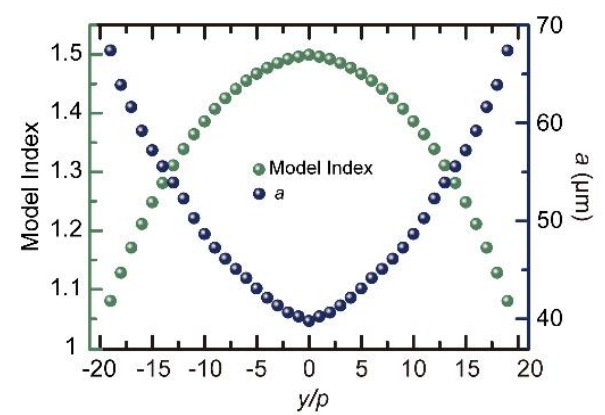

Figure 1. (a) Schematic of subwavelength metallic pillar on a metallic slab with height $h=70 \mu \mathrm{m}$ and period $p=80 \mu \mathrm{m}$. (b) Dispersion relation of THz SSPPs for metal pillars with different lateral widths. (c) Retrieved mode index for SSPPs at $0.73 \mathrm{THz}$ with lateral width a varied from $34 \mu \mathrm{m}$ to $76 \mu \mathrm{m}$ with a step of $2 \mu \mathrm{m}$. (d) Required mode index for SSPP GRIN lens and lateral widths of corresponding selected pillars.

In order to verify the feasibility of the proposed scheme, a GRIN lens for THz SSPPs was demonstrated as a proof of the concept. Compared with classical lenses, GRIN lenses have the advantage that they can be flat and free of geometrical aberrations. In our design, the twodimensional GRIN lens has a uniform index distribution along the $x$-direction and a gradient index distribution along the $y$-direction: ${ }^{44}$

$$
n(y)=n_{0} \sqrt{1-\alpha^{2} y^{2}}, \alpha=\frac{1}{D} \sqrt{\left(\frac{n_{D}}{n_{0}}\right)},(|y| \leq D),
$$

where $n_{0}$ and $n_{D}$ are the maximum and minimum indices at the center and edge of the GRIN 
lens, respectively; $\alpha$ is a parameter defining the curvature of the index parabola; and $D$ is the half width in the $y$ direction of the lens. From the ray-optics point of view, such a GRIN lens collimates any paraxial input ray at the characteristic distance (i.e., the focal length) $L=\pi / 2 \alpha$. By setting $n_{0}$ $=1.5, n_{D}=1.06$, and $D=1520 \mu \mathrm{m}$, the required index distribution along the $y$-direction and the lateral widths of the corresponding selected pillars are illustrated in Figure 1d as the green and blue dots, respectively.

To experimentally verify our proposed design, we fabricated the sample using conventional photolithography, deep reactive ion etching, and metallization processing. A momentum-matched metacoupler made of C-shaped-slit resonators (CRs) was used to efficiently convert free-space THz radiation to SSPPs at $0.73 \mathrm{THz}{ }^{45}$ Figure 2a shows the schematic of a single CR with the geometrical parameters of radius $r$, width $w$, open angle $\theta$, and orientation angle $\gamma$. It has been demonstrated that such resonators can produce phase retardation for the cross-polarization output over the entire $2 \pi$ phase range, and the conversion from normal incident plane waves to surface waves can be achieved by properly arranging the phase gradient. ${ }^{45}$ In our design, five different CRs with a super period $\Lambda=400 \mu \mathrm{m}$ were chosen to satisfy the phase gradient $\mathrm{d} \varphi / \mathrm{d} x=k_{\mathrm{SP}}$, where Fig. $2 \mathrm{~b}$ illustrates part of the fabricated metacoupler. The measured time-domain and frequencydomain signals on the top of the metacoupler are illustrated in Figs. 2c and 2d, respectively, indicating that the SSPPs were successfully excited with a center frequency around $0.73 \mathrm{THz}$. Here the conversion efficiency of the proposed coupler cannot be directly measured in the experiments currently. Alternatively, it can be estimated in the simulation by integrating the electric-field intensity over an area above the metal surface, and is approximately $23.3 \%{ }^{45}$ 
(a)

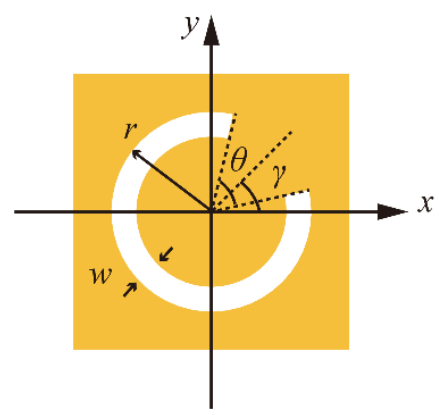

(c)

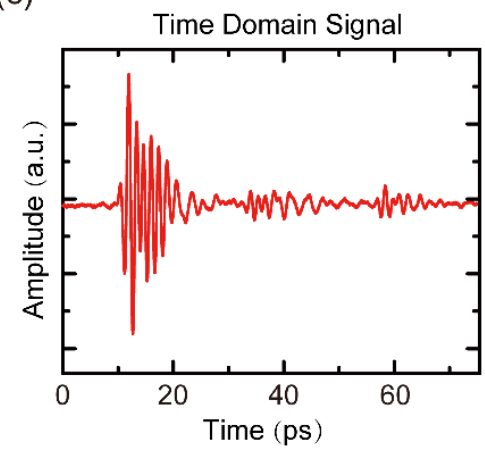

(b)

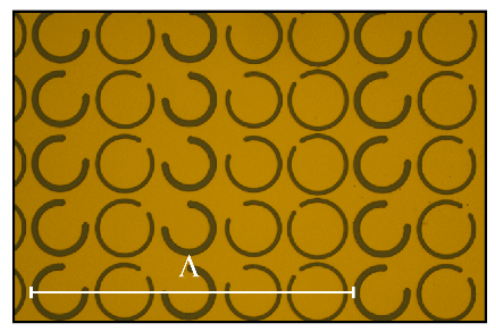

(d)

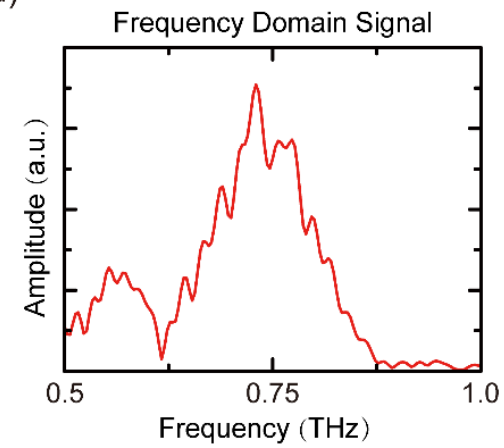

Figure 2. (a) Schematic of a single CR, where the periods along $x$ and $y$ directions are both $80 \mu \mathrm{m}$. (b) Microscopy image of fabricated metacoupler, where the parameters of the CRs within a super period are: radius $r=36,37,35,36,38 \mu \mathrm{m}$, width $w=9,6,10,7,5 \mu \mathrm{m}$, open angle $\theta=65^{\circ}, 17^{\circ}$, $80^{\circ}, 43^{\circ}, 10^{\circ}$, and orientation angle $\gamma=45^{\circ}, 45^{\circ}, 135^{\circ}, 135^{\circ}, 135^{\circ}$, respectively, from left to right. (c, d) Measured time-domain and frequency-domain signals in the excitation range, respectively.

Figure $3 \mathrm{a}$ illustrates the schematic of the sample and the measurement setup, where the total area of the GRIN lens was about $5.0 \times 3.2 \mathrm{~mm}^{2}$. The inset at the bottom-right corner illustrates the enlarged scanning electron microscopy (SEM) image of part of the fabricated GRIN lens, whose mode index distribution is shown in Figure 3b. Figure 3c illustrates the simulated SSPP fields in the excitation range $(x<0)$ and on the GRIN lens $(x>0)$, respectively, which were obtained by setting electric field-monitors in the $x y$-plane that was $100 \mu \mathrm{m}$ above the pillar structures. Clearly, the well-confined SSPP fields were gradually varied from a plane wave to a converging wave when 
passing through the GRIN lens, which was eventually focused to a point in the Fourier plane along the $x$-direction. Then, the wavefront of the SSPPs turned into divergence behind the focus, which complies with the periodic wavefront variation of the GRIN lens design. An SNTM was used to experimentally measure the SSPP fields, as schematically shown in Figure 3a. The THz beam generated from a photoconductor antenna was illuminated on the backside of the metacoupler to excite the SSPPs, and the plasmonic fields were detected by a near-field probe above the pillar arrays at a distance about $100 \mu \mathrm{m}$ (see Section 3 of the Supporting Information for the details about the SNTM and measurements). The propagating SSPP fields on the GRIN lens were scanned in a range of $3.5 \times 3 \mathrm{~mm}^{2}$, as shown in Figure $3 \mathrm{~d}$. It can be seen that the measured results agree well with the simulation results, which verified the proposed design scheme. Figure $3 \mathrm{e}$ illustrates the extracted normalized amplitude in Figure $3 \mathrm{~d}$ as a function of the $y$ coordinate at the focus, which shows a full width at half-maximum of about $410 \mu \mathrm{m}$. 
(a)

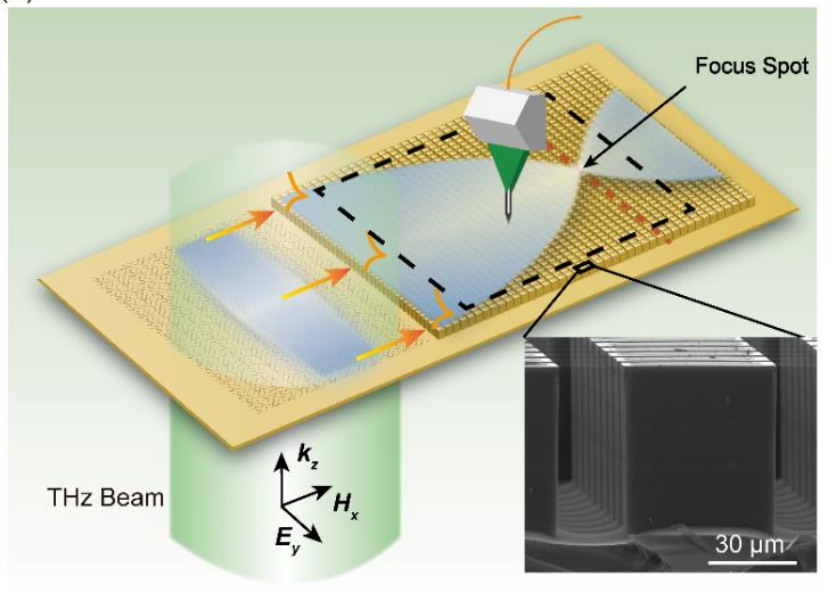

(b)

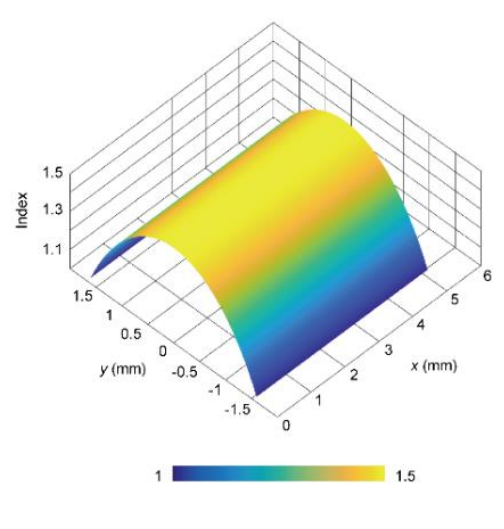

(c)

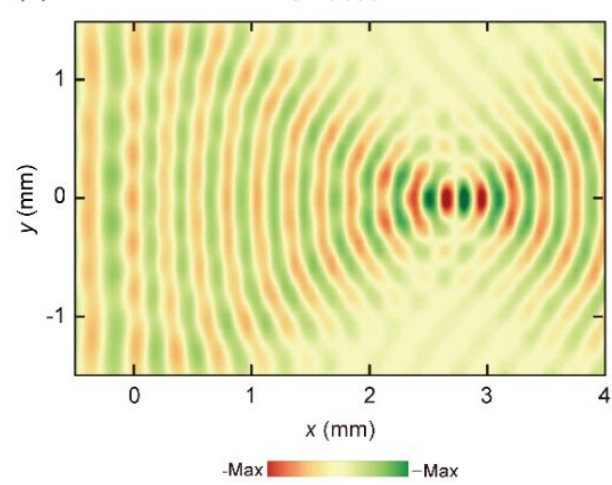

(d)

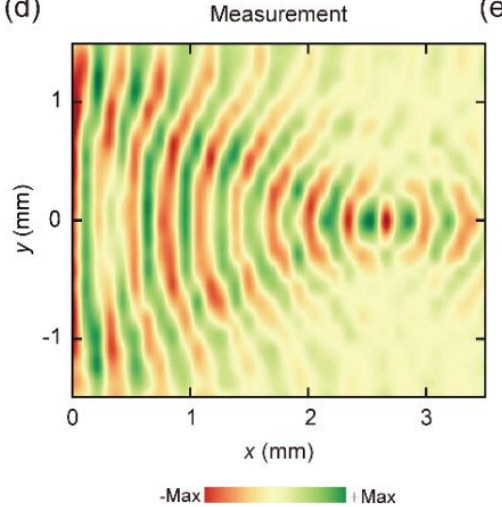

(e) Amplitude (a.u.)

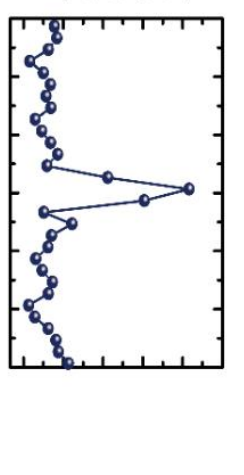

Figure 3. (a) Full schematic of the experimental setup, where the inset SEM image illustrates part of the fabricated sample. (b) The index distribution of fabricated GRIN lens. (c) Simulated and (d) measured near electric-field distributions at $0.73 \mathrm{THz}$ on the observation plane. (e) Measured normalized $E_{\mathrm{z}}$-field amplitude along the $y$ direction at the focus, showing the horizontal confinement.

To further exploit the versatility of proposed design scheme, we designed and characterized three kinds of integrated devices: SSPP telescope, SSPP waveguide coupler, and SSPP multiplexer. The telescope was combined by two different GRIN lenses, as shown by the SEM image in Figure 4a. Here, the half widths of these two GRIN lenses were $D_{1}=1520 \mu \mathrm{m}$ and $D_{2}=720 \mu \mathrm{m}$, respectively, and the length of each GRIN lens along the $x$-direction was defined by its own collimation distance. These two GRIN lenses formed a flat telescope in confocal arrangement, 
where the periphery range was filled by unit cells with the minimum mode index. Figure $4 \mathrm{~b}$ illustrates the simulation results, where the excited SSPPs travel across the telescope from the larger GRIN lens side horizontally. It is clearly seen that the incident flat-wavefront SSPPs with a width about $2 D_{1}$ varied from being concave to convex, and were eventually transformed into flatwavefront SSPPs with a width about $2 D_{2}$. Likewise, when the SSPPs were incident from the smaller GRIN lens side, their scale was accordingly magnified by $D_{1} / D_{2}$ times. The measured real part of the $E_{\mathrm{z}}$-field distribution shown in Figure 4c agrees well with simulations, showing a compressed flat wavefront at the output port that was different from the diverging wavefront in Fig. 3d. Both simulation and experimental results verify the wavefront scaling capability of the proposed SSPP telescope. 
(a)

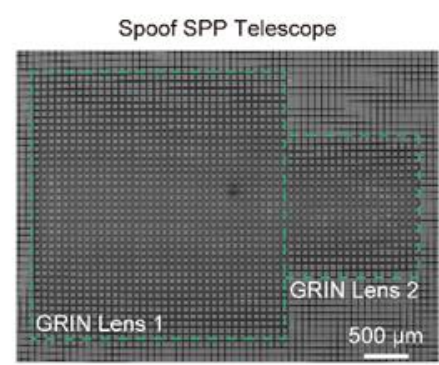

(d)

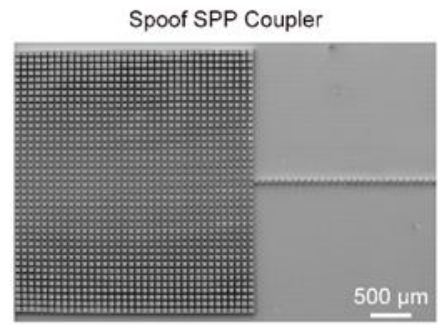

(g)

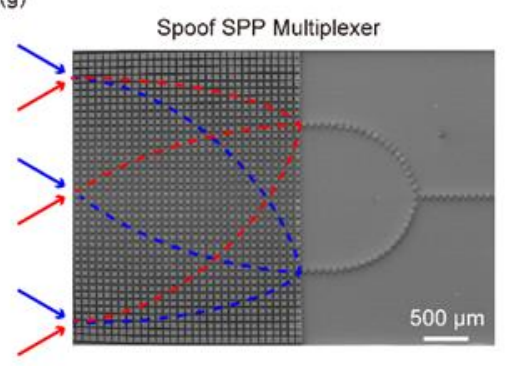

(b)

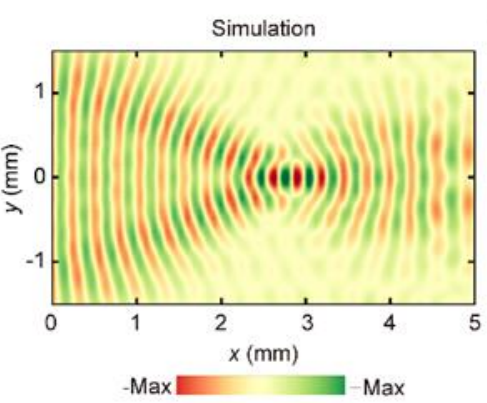

(e)

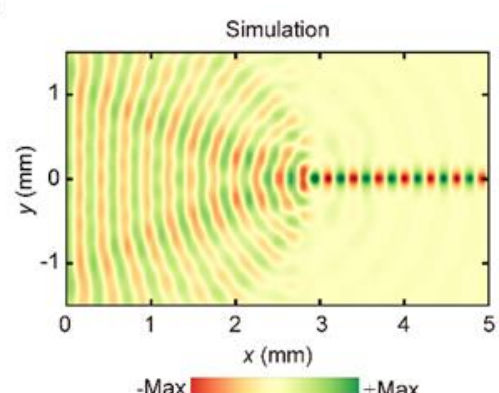

(h)

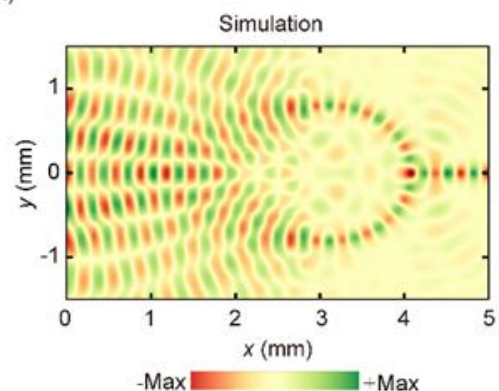

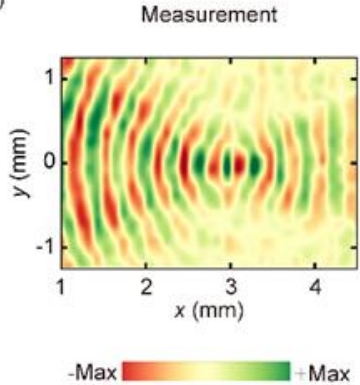

(f)

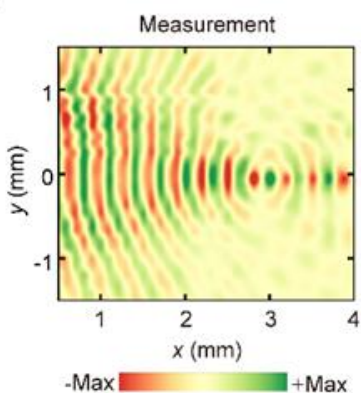

(i)

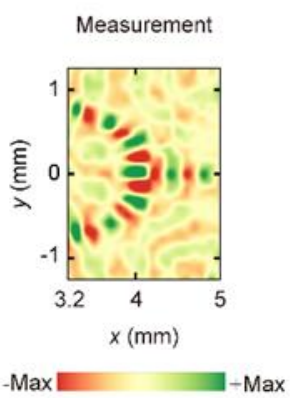

Figure 4. (a, d, g) SEM images of the fabricated SSPP telescope sample, SSPP coupler sample, and the SSPP multiplexer, respectively. (b, e, h) Simulated real parts of $E_{\mathrm{z}}$-field distribution at 0.73 $\mathrm{THz}$, respectively. (c, f, i) Measured real parts of $E_{\mathrm{z}}$-field distribution at $0.73 \mathrm{THz}$ using SNTM with a scanning range of $3.5 \times 2.5 \mathrm{~mm}^{2}, 3.5 \times 3 \mathrm{~mm}^{2}$, and $1.8 \times 2.5 \mathrm{~mm}^{2}$, respectively.

In free-space, the GRIN lens is frequently used as a fiber coupling element. So far, most of the reported $\mathrm{THz}$ plasmonic couplers functionally convert free-space $\mathrm{THz}$ beams to plasmonic waves with a certain width of the wavefront. ${ }^{42,43}$ For designing ultra-compact plasmonic circuits, it is highly desired to couple SSPPs into a single waveguide. We fabricated an SSPP waveguide coupler that combined an SSPP GRIN lens and a straight SSPP waveguide, as illustrated in Figure 
4d. The width and the index distribution along the $y$-direction of this GRIN lens are the same as those in Figure 3, and meanwhile the length along the $x$-direction was set as its collimation distance. The straight waveguide was comprised of the same periodic unit cells as the central ones in the GRIN lens, ensuring a smooth impedance matching. Figure 4e illustrates the simulation results, where the flat-wavefront SSPPs were successfully focused at the interface center between the GRIN lens and the waveguide, and then were coupled into and propagated along the straight waveguide. The corresponding measurements agree well with simulations, as shown in Figure 4f, experimentally confirming the viability of the SSPP wavegudie coupler. In addition to the proposed design, such a coupling scheme could smoothly link different efficient THz metacouplers ${ }^{45,46}$ and diverse plasmonic waveguides, ${ }^{30-34}$ which may motivate the design and fabrication of future practical plasmonic circuits.

Since the GRIN lens could focus different oblique incidences at the same collimation distance with the focal points shifted along the direction perpendicular to the optical axis, it is possible to design a multiplexer for obliquely incident SSPPs based on the proposed design scheme. Figure 4g illustrates the SEM image of an SSPP multiplexer, which is composed of an SSPP GRIN lens and a Y-shaped SSPP waveguide. In order to get obliquely incident SSPPs, two sets of crossed periodic gratings were utilized as the metacoupler to convert incident free-space $\mathrm{THz}$ beams to two SSPP excitations with angles of $30^{\circ}$ and $-30^{\circ}$ with respect to the $x$-axis (see Section 4 of Supporting Information for the detailed design and corresponding simulation results). The two endpoints of the Y-shaped waveguide were connected with the GRIN lens at the focal points of the obliquely incident SSPPs, as schematically shown by the dashed lines in Figure 4g. The simulated real part of the $E_{\mathrm{z}}$-field distribution was shown in Figure $4 \mathrm{~h}$, where two obliquely incident SSPP excitations were focused at the corresponding endpoints of the Y-shaped waveguide, and were then 
multiplexed into and propagated along the straight part of the waveguide. The SSPP fields at the multiplexing points were scanned using SNTM, as shown in Figure 4i, which agree well with simulations and experimentally verify the proposed SSPP multiplexer. Interestingly, if the SSPPs were incident from the straight waveguide side, this device could function as a splitter, i.e., the incident SSPPs would split into two SSPP beams at the left end of the GRIN lens with angles of $30^{\circ}$ and $-30^{\circ}$ with respect to the $x$-axis. Furthermore, by removing the straight waveguide, this device could function as a reflector to reflect the $30^{\circ}$ incident SSPPs to $-30^{\circ}$ output SSPPs, or vice versa.

The presented GRIN device design scheme extends the utilization of metallic pillars from simple waveguiding to refractive index manipulation, making SSPPs easier to be manipulated and utilized; meanwhile, the cascadable feature of the proposed GRIN devices would be quite practical for designing miniaturized and multifunctional on-chip $\mathrm{THz}$ system. Firstly, future wireless communication networks need to handle data rates of tens or even hundreds of Gbit/s per link, which requires carrier frequencies in the unallocated $\mathrm{THz}$ regime. The presented GRIN devices could serve as a data stream bridge between the wireless signals and on-chip microprocessors, since they can flexibly couple free-space THz waves into SSPPs with diverse functionalities, i.e., focusing, multiplexing, and routing to desired positions or components. Secondly, the strong field confinement of the SSPPs can enable enhanced light-matter interaction, which has great quite potential for on-chip sensing applications. For instance, the proposed SSPP multiplexer could also function as a Mach-Zehnder interferometer, where the change in the dielectric environment around one interferometer arm could be revealed by the interference spectra. We envision that the ensuing studies of such complex GRIN devices and integrated on-chip systems could motivate and enrich various practical applications. 


\section{Conclusion}

We have experimentally demonstrated the feasibility of tailoring the mode index of $\mathrm{THz}$ SSPPs by metallic pillars. The proposed variable refractive index structures would therefore serve as basic elements for designing THz SSPP devices. We have verified our approach with some integrated GRIN devices that can flexibly manipulate the propagation of well confined THz SSPPs. In addition to the exhibited slowly varied index distributions, our approach could also achieve abrupt or arbitrary index distributions. Such flexibility in conjunction with the recently reported

computational inverse-design approaches ${ }^{47,48}$ - algorithms for discovering index distributions to achieve desired functions - would realize various plasmonic devices with extreme performance, contributing to the development of miniaturized and multifunctional on-chip THz systems.

\section{Supporting Information}

This material is available free of charge at http://pubs.acs.org.

There are four sections:

1). Description of sample fabrication;

2) Simulation details;

3). Method of SNTM experimental system and characterization;

4). Oblique launcher.

\section{AUTHOR INFORMATION}

\section{Corresponding Authors}

*Email: quanxu@tju.edu.cn.

*Email: jiaghan@tju.edu.cn.

*Email: weili.zhang@okstate.edu. 


\section{Notes}

The authors declare no competing financial interest.

\section{ACKNOWLEDGEMENT}

This work was funded by National Natural Science Foundation of China $(61805129,11874245$, 61875150, 61935015, 11974259, 61775159, 62005193, 62075158); Applied Basic Research Project of ShanXi Province (201801D221161); Key Research and Development Program of Shanxi Province (201903D121026, 201903D121071); Applied Basic Research Project of Datong City (2018148); China Postdoctoral Science Foundation (2020TQ0224); Tianjin Municipal Fund for Distinguished Young Scholars (18JCJQJC45600); and KAUST Office of Sponsored Research (URF-2950-CRG5).

\section{References}

1. Tonouchi, M. Cutting-edge terahertz technology. Nat. photonics, 2007, 1, 97-105.

2. Kawano, Y.; Ishibashi, K. An on-chip near-field terahertz probe and detector. Nat. Photonics, 2008, 2, 618-621.

3. Saeedkia, D. Handbook of terahertz technology for imaging, sensing and communications; Woodhead Publishing Limited, 2013, DOI 10.1533/9780857096494.

4. Zhang, X.; Xu, Q.; Xia, L.; Li, Y.; Gu, J.; Tian, Z.; Ouyang, C.; Han, J.; Zhang, W. Terahertz surface plasmonic waves: a review. Adv. Photonics 2020, 2, 014001. 
5. Maier, S. A. Plasmonics: fundamentals and applications; Springer, 2007, DOI $10.1007 / 9780387378251$.

6. Tanemura, T.; Balram, K. C.; Ly-Gagnon, D.; Wahl, P.; White, J. S.; Brongersma, M. L.; Miller, D. A. B. Multiple-wavelength focusing of surface plasmons with a nonperiodic nanoslit coupler. Nano Lett. 2011, 11, 2693-2698.

7. Spektor, G.; David, A.; Gjonaj, B.; Bartal, G.; Orenstein, M. Metafocusing by a metaspiral plasmonic lens. Nano lett. 2015, 15, 5739-5743.

8. Wintz, D.; Genevet, P.; Ambrosio, A.; Woolf, A.; Capasso, F. Holographic metalens for switchable focusing of surface plasmons. Nano lett. 2015, 15, 3585-3589.

9. Wei, H.; Wang, Z.; Tian, X.; Käll, M.; Xu, H. Cascaded logic gates in nanophotonic plasmon networks. Nat Commun. 2011, 2, 387.

10. Fu, Y.; Hu, X.; Lu, C.; Yue, S.; Yang, H.; Gong, Q. All-optical logic gates based on nanoscale plasmonic slot waveguides. Nano lett. 2012, 12, 5784-5790.

11. Huang, L.; Chen, X.; Bai, B.; Tan, Q.; Jin, G.; Zentgraf, T.; Zhang, S. Helicity dependent directional surface plasmon polariton excitation using a metasurface with interfacial phase discontinuity. Light Sci. Appl. 2013, 2, e70.

12. Lin, J.; Mueller, J. P. B.; Wang, Q.; Yuan, G.; Antoniou, N.; Yuan, X.; Capasso, F. Polarizationcontrolled tunable directional coupling of surface plasmon polaritons. Science, 2013, 340, $331-334$.

13. Xiao, S.; Zhong, F.; Liu, H.; Zhu, S.; Li, J. Flexible coherent control of plasmonic spin-Hall effect. Nat Commun. 2015, 6, 8360.

14. Ding, F.; Deshpande, R.; Bozhevolnyi, S. I. Bifunctional gap-plasmon metasurfaces for visible light: polarization-controlled unidirectional surface plasmon excitation and beam steering at 
normal incidence. Light Sci. Appl. 2018, 7, 17178.

15. Sorger, V. J.; Oulton, R. F.; Ma, R. M.; Zhang, X. Toward integrated plasmonic circuits. MRS bull, 2012, 37, 728-738.

16. Guo, X.; Ma, Y.; Wang, Y.; Tong, L. Nanowire plasmonic waveguides, circuits and devices. Laser \& Photon. Rev. 2013, 7, 855-881.

17. Fang, Y.; Sun, M. Nanoplasmonic waveguides: towards applications in integrated nanophotonic circuits. Light Sci. Appl. 2015, 4, e294.

18. Pendry, J. B.; Martin-Moreno, L.; Garcia-Vidal, F. J. Mimicking surface plasmons with structured surfaces. Science 2004, 305, 847-848.

19. Hibbins, A, P.; Evans, B. R.; Sambles, J. R. Experimental verification of designer surface plasmons. Science 2005, 308, 670-672.

20. Gao, Z.; Wu, L.; Gao, F.; Luo, Y.; Zhang, B. Spoof plasmonics: from metamaterial concept to topological description. Adv. Mater. 2018, 30, 1706683.

21. Zhang, H. C.; Zhang. L. P.; He, P. H.; Xu, J.; Qian, C.; Garcia-Vidal, F. J.; Cui, T. J. A plasmonic route for the integrated wireless communication of subdiffraction-limited signals. Light Sci. Appl. 2020, 9, 113.

22. Shen, X.; Cui, T. J.; Martin-Cano, D.; Garcia-Vidal, F. J. Conformal surface plasmons propagating on ultrathin and flexible films. Proc. Natl. Acad. Sci. USA 2013, 110, 40-45.

23. Ma, H.; Shen, X.; Cheng, Q.; Jiang, W.; Cui, T. J. Broadband and high - efficiency conversion from guided waves to spoof surface plasmon polaritons. Laser \& Photon. Rev. 2014, 8, 146151.

24. Zhang, H. C.; Liu, S.; Shen, X.; Chen, L. H.; Li, L.; Cui, T. J. Broadband amplification of spoof surface plasmon polaritons at microwave frequencies. Laser \& Photon. Rev. 2015, 9, 
83-90.

25. Yu, N.; Wang, Q. J.; Kats, M. A.; Fan, J. A.; Khanna, S. P.; Li, L.; Davies, A. G.; Linfield, E. H.; Capasso, F. Designer spoof surface plasmon structures collimate terahertz laser beams. Nat. Mater. 2010, 9, 730-735.

26. Ng, B.; Wu, J.; Hanham, S. M.; Fernández-Domínguez, A. I.; Klein, N.; Liew, N. F.; Breese, M. B. H.; Hong, M.; Maier, S. A. Spoof plasmon surfaces: a novel platform for THz sensing. Adv. Opt. Mater. 2013, 1, 543-548.

27. Ng, B.; Hanham, S. M.; Wu, J.; Fernández-Domínguez, A. I.; lein, N.; Liew, Y. F.; Breese, M. B. H.; Hong, M.; Maier, S. A. Broadband terahertz sensing on spoof plasmon surfaces. ACS Photon. 2014, 1, 1059-1067.

28. Pan, B. V.; Liao, Z.; Zhao, J.; Cui, T. J. Controlling rejections of spoof surface plasmon polaritons using metamaterial particles. Opt. Express 2014, 22, 13940-13950.

29. Yin, J. Y.; Ren, J.; Zhang, H. C.; Pan, B. C.; Cui, T. J. Broadband frequency-selective spoof surface plasmon polaritons on ultrathin metallic structure. Sci. Rep. 2015, 5, 8165.

30. Fernández-Domínguez, A. I.; Moreno, E.; Martín-Moreno, L.; García-Vidal, F. J. Guiding terahertz waves along subwavelength channels. Phys. Rev. B 2009, 79, 233104.

31. Fernández-Domínguez, A. I.; Moreno, E.; Martín-Moreno, L.; García-Vidal, F. J. Terahertz wedge plasmon polaritons. Opt. Lett. 2009, 34, 2063-2065.

32. Kumar, G.; Li, S.; Jadidi, M. M.; Murphy, T. E. Terahertz surface plasmon waveguide based on a one-dimensional array of silicon pillars. New J. Phys. 2013, 15, 085031.

33. Zhang, Y.; Xu, Y.; Tian, C.; Xu, Q.; Zhang, X.; Li, Y.; Zhang, X.; Han, J.; Zhang, W. Terahertz spoof surface-plasmon-polariton subwavelength waveguide. Photonics Res. 2018, 6, 18-23.

34. Yuan, M.; Li, Y.; Lu, Y.; Zhang, Y.; Zhang, Z.; Zhang, X.; Zhang, X.; Han, J.; Zhang, W. High- 
performance and compact broadband terahertz plasmonic waveguide intersection. Nanophotonics 2019, 8, 1811-1819.

35. Huidobro, P. A.; Nesterov, M. L.; Martín-Moreno, L.; García-Vidal, F. J. Transformation optics for plasmonics. Nano Lett. 2010, 10, 1985-1990.

36. Liu, Y.; Zentgraf, T.; Bartal, G.; Zhang, X. Transformational plasmon optics. Nano Lett. 2010, 10, 1991-1997.

37. Zentgraf, T.; Liu, Y.; Mikkelsen, M. H.; Valentine, J.; Zhang, X. Plasmonic luneburg and eaton lenses. Nat. Nanotech. 2011, 6, 151-155.

38. Wu, Q.; Turpin, J. P.; Werner, D. H. Integrated photonic systems based on transformation optics enabled gradient index devices. Light. Sci. Appl. 2012, 1, e38.

39. Zhao, C.; Liu, Y.; Zhao, Y.; Fang, N.; Huang, T. J. A reconfigurable plasmofluidic lens. Nat. Commun. 2013, 4, 2305.

40. Zhang, J.; Pendry, J. B.; Luo, Y. Transformation optics from macroscopic to nanoscale regimes: a review. Adv. Photon. 2019, 1, 014001.

41. Renger, J.; Kadic, M.; Dupont, G.; Aćimović, S. S.; Guenneau, S.; Quidant, R.; Enoch, S. Hidden progress: broadband plasmonic invisibility. Opt. Express 2010,18, 15757-15768.

42. Kadic, M.; Guenneau, S.; Enoch, S.; Huidobro, P. A.; Martín-Moreno, L.; García-Vidal, F. J.; Renger, J.; Quidant, R. Transformation plasmonics. Nanophotonics 2012, 1, 51-64.

43. Wan, X.; Shen, X.; Luo, Y.; Cui, T. J. Planar bifunctional Luneburg - fisheye lens made of an anisotropic metasurface. Laser \& Photon. Rev. 2014, 8, 757-765.

44. Mendlovic, D. and Ozaktas, H. M., Fractional Fourier transforms and their optical implementation: I, J. Opt. Soc. Am. A. 1993, 10, 1875-1881.

45. Xu, Q.; Zhang, X.; Wei, M.; Ren, G.; Xu, Y.; Li, Y.; Wang, H.; Ouyang, C.; Han, J.; Zhang, 
W. Efficient metacoupler for complex surface plasmon launching. Adv. Opt. Mater. 2018, 6, 1701117.

46. Liu, S.; Zhang, H. C.; Zhang, L.; Yang, Q. L.; Xu, Q.; Gu, J.; Yang, Y.; Zhou, X. Y.; Han, J.; Cheng, Q.; Zhang, W.; Cui, T. J. Full-state controls of terahertz waves using tensor coding metasurfaces. ACS Appl. Mater. Interfaces 2017, 9, 21503-21514.

47. Molesky, S.; Lin, Z.; Piggott, A. Y.; Jin, W.; Vucković, J.; Rodriguez, A. W. Inverse design in nanophotonics. Nat. Photon. 2018, 12, 659-670.

48. Yao, K.; Unni, R.; Zheng, Y. Intelligent nanophotonics: merging photonics and artificial intelligence at the nanoscale. Nanophotonics 2019, 8, 339-366. 